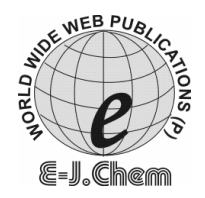

http://www.e-journals.net
ISSN: 0973-4945; CODEN ECJHAO

E-Journal of Chemistry 2010, 7(3), 935-941

\title{
Synthesis and In Vitro Antimicrobial, Anthelmintic and Insecticidal Activities Study of 4(4'-Bromophenyl)-6-substituted-aryl-1-acetyl pyrimidine-2-thiols
}

\author{
RITA BAMNELA* and S. P. SHRIVASTAVA \\ *Department of Chemistry, Govt. P.G. College, \\ Damoh, M.P. -470 661, India. \\ Synthetic Organic Laboratory, Department of Chemistry, \\ Dr. Hari Singh Gour, University, Sagar - 470 003, M.P, India. \\ ritabamnela@gmail.com
}

Received 7 January 2010; Accepted 5 March 2010

\begin{abstract}
A new series of 4(4'-bromophenyl)-6-substituted aryl-1-acetyl pyrimidine-2-thiol derivatives were synthesized by heating chalcones with thiourea, in the presence of ethanolic potassium hydroxide, followed by treatment with acetyl chloride. The structure of the compounds was characterized by IR and $\mathrm{H}^{1}$ NMR spectral study and elemental analysis. The compounds were screened for their antimicrobial, anthelmintic and insecticidal activities. All the compounds exhibited significant to moderate biological activities.
\end{abstract}

Keywords: Pyrimidine-2-thiols, Antimicrobial, Anthelmintic, Insecticidal activity.

\section{Introduction}

Heterocyclic system containing pyrimidine ring, constitute an important class of potentially bio-active compounds. The earlier workers reported that, pyrimidine derivatives are associated with a wide spectrum of biological activities viz. antimicrobial ${ }^{1-4}$, anthelmintic ${ }^{5-7}$, anticancer ${ }^{8-9}$, antitumer $^{10}$, anti-inflammatory ${ }^{11-12}$, anti-HIV agents ${ }^{13}$, calcium-channel-blocker ${ }^{14}$ and insecticidal agents ${ }^{15}$ and this significance led us to synthesize the titled compounds.

In the present communication, we have reported, the synthesis of a new series of 4(4'bromophenyl)-6-substituted aryl-1-acetyl pyrimidine-2-thiols (Scheme 1) and evaluated for their antimicrobial, anthelmintic and insecticidal activities. 


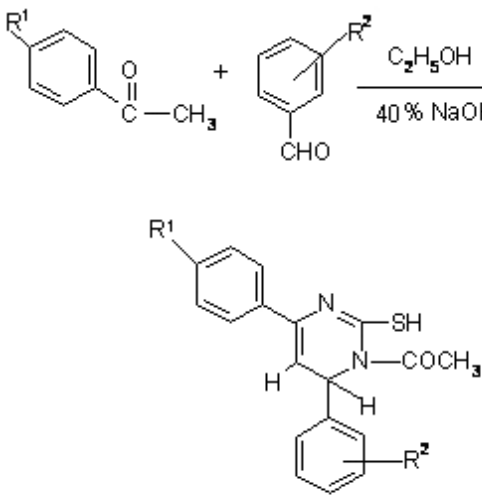

[3] $(a-j)$
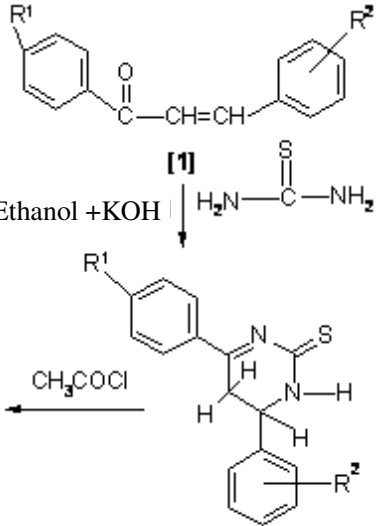

[2]

Where - $\mathrm{R}^{1}=$ Bromo, $\mathrm{R}^{2}=4-\mathrm{O}-\mathrm{CH}_{3}, 4-\mathrm{OH}-3-\mathrm{OCH}_{3}, \mathrm{H}, 4-\mathrm{Cl}, 2-\mathrm{Cl}, 4-\mathrm{N}\left(\mathrm{CH}_{3}\right)_{2}, 2-\mathrm{NO}_{2}, 3-$ $\mathrm{NO}_{2}$, Furyl, 2-OH.

\section{Experimental}

\section{Scheme 1}

The melting points were recorded by open capillary method and are uncorrected. IR spectra $\left(\boldsymbol{V}_{\max }\right.$ in $\left.\mathrm{cm}^{-1}\right)$ were recorded on a Shimadzu FTIR 8300 spectrophotometer, using $\mathrm{KBr}$ pellets. The $\mathrm{H}^{1}$ NMR spectra were recorded on a DRX-300 (300 MHz) instrument using $\mathrm{CDCl}_{3}$ as solvent (Chemical Shift in $\delta \mathrm{ppm}$ ), using TMS as internal standard. The completion of reactions was monitored by TLC.

\section{General procedure}

Synthesis of 4-bromochalcones (1a-j)

4-Bromoacetophenone $(0.01 \mathrm{~mol})$ and anisaldehyde $(0.01 \mathrm{~mol})$ were stirred in ethanol $(20 \mathrm{~mL})$ and to it $10 \mathrm{~mL}$ of $40 \% \mathrm{NaOH}$ solution was added. The mixture was kept overnight at room temperature. The contents were then poured on crushed ice and acidified with dil. $\mathrm{HCl}$. The solid was filtered, dried and recrystallised from ethanol. The other chalcones were prepared with the same procedure using different aromatic aldehydes.

4(4'-Bromophenyl)-6-substituted phenyl-1, 5, 5, 6-tetrahydropyrimidine-2-thiones (2a-j)

The chalcones $(0.01 \mathrm{~mol})$, thiourea $(0.01 \mathrm{~mol})$ and $\mathrm{KOH}(1.0 \mathrm{~g})$ in ethanol $(20 \mathrm{~mL})$ was refluxed on water bath for $6 \mathrm{~h}$ and completion of reaction was monitored by TLC. After keeping in icecold condition, the solid was collected and recrystallised from ethanol.

Yield $71 \%$, m.p. $206^{\circ} \mathrm{C}$, m.wt.374.9, (\% of C : found 54.38 - calculated 54.41$)$, (\% of $\mathrm{H}$ : found 03.98 , calculated 4.00$),\left(\%\right.$ of $\mathrm{N}$ : found 7.40 , calculated 7.46), IR $(\mathrm{KBr})$ in cm ${ }^{-1}$ : 536-600 (Ar-Br str), 1595-1560 (C=N str), 1220-1200 (C=S str.), 820-740 (Ar-H def. pyrimidine ring breath), 3351-3300 (N-H Str). The other derivatives were prepared with different chalcones, applying the same procedure.

4(4'-Bromophenyl)-6-substitutedphenyl-1-acetyl-5, 6-dihydropyrimidine-2-thiols (3a-j) The different thiones $(\mathbf{2 a - j}),(0.005 \mathrm{~mol})$ and acetyl chloride $(16 \mathrm{~mL})$ were heated at $30-40{ }^{\circ} \mathrm{C}$, under reflux on water bath for $2 \mathrm{~h}$, completion of reaction was monitored by TLC. Excess of acetyl chloride was evaporated and the oily product obtained, was treated with pet ether. The solid obtained was filtered off, dried and recrystallised from ethanol. The physical and spectral data of all the synthesized compounds described as follows: 
Table 1. Physical data of compounds 3a-j.

\begin{tabular}{|c|c|c|c|c|c|c|c|c|c|}
\hline \multirow{2}{*}{$\begin{array}{l}\dot{0} \\
\dot{z} \\
\dot{0} \\
0\end{array}$} & \multirow[b]{2}{*}{$\mathrm{R}^{2}$} & \multirow[b]{2}{*}{ M.F. } & \multirow[b]{2}{*}{ M.wt. } & \multirow[b]{2}{*}{$\begin{array}{c}\text { M.P. } \\
{ }^{\circ} \mathrm{C}\end{array}$} & \multirow[b]{2}{*}{ 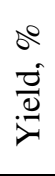 } & \multicolumn{4}{|c|}{ Elemental Analysis \% } \\
\hline & & & & & & & $\mathrm{C}$ & $\mathrm{H}$ & $\mathrm{N}$ \\
\hline \multirow{2}{*}{$\mathbf{3 a}$} & \multirow{2}{*}{$4-\mathrm{O}-\mathrm{CH}_{3}$} & \multirow{2}{*}{$\mathrm{C}_{19} \mathrm{H}_{17} \mathrm{O}_{2} \mathrm{~N}_{2} \mathrm{SBr}$} & \multirow{2}{*}{416.9} & \multirow{2}{*}{215} & \multirow{2}{*}{85} & found & 54.62 & 4.05 & 6.69 \\
\hline & & & & & & calc. & 54.68 & 4.07 & 6.71 \\
\hline \multirow{2}{*}{$3 \mathbf{b}$} & \multirow{2}{*}{$4-\mathrm{OH}-3-\mathrm{OCH}_{3}$} & \multirow{2}{*}{$\mathrm{C}_{19} \mathrm{H}_{17} \mathrm{O}_{3} \mathrm{~N}_{2} \mathrm{SBr}$} & \multirow{2}{*}{432.9} & \multirow{2}{*}{185} & \multirow{2}{*}{86} & found & 52.65 & 3.90 & 6.42 \\
\hline & & & & & & calc. & 52.66 & 3.92 & 6.46 \\
\hline \multirow{2}{*}{$3 \mathbf{c}$} & \multirow{2}{*}{$\mathrm{H}$} & \multirow{2}{*}{$\mathrm{C}_{18} \mathrm{H}_{15} \mathrm{~N}_{2} \mathrm{OSBr}$} & \multirow{2}{*}{386.9} & \multirow{2}{*}{218} & \multirow{2}{*}{65} & found & 55.80 & 3.82 & 7.20 \\
\hline & & & & & & calc. & 55.82 & 3.87 & 7.23 \\
\hline \multirow{2}{*}{ 3d } & \multirow{2}{*}{$4-\mathrm{Cl}$} & \multirow{2}{*}{$\mathrm{C}_{18} \mathrm{H}_{14} \mathrm{~N}_{2} \mathrm{OSClBr}$} & \multirow{2}{*}{421.4} & \multirow{2}{*}{194} & \multirow{2}{*}{75} & found & 51.21 & 3.30 & 6.61 \\
\hline & & & & & & calc. & 51.25 & 3.32 & 6.64 \\
\hline \multirow{2}{*}{$3 \mathbf{e}$} & \multirow{2}{*}{$2-\mathrm{Cl}$} & $\mathrm{C}_{18} \mathrm{H}_{14} \mathrm{~N}_{2} \mathrm{OSClBr}$ & 421.4 & 220 & 70 & found & 51.20 & 3.30 & 6.60 \\
\hline & & $C_{18} \Pi_{14} \mathrm{IV}_{2}$ USCIDI & 421.4 & 220 & 10 & calc. & 51.25 & 3.32 & 6.64 \\
\hline 3f & $4-\mathrm{N}\left(\mathrm{CH}_{3}\right)_{2}$ & $\mathrm{C}_{20} \mathrm{H}_{20} \mathrm{~N}_{3} \mathrm{OSBr}$ & 429.9 & 200 & 72 & found & 55.80 & 4.61 & 9.72 \\
\hline & & & & & 12 & calc. & 55.82 & 4.65 & 9.76 \\
\hline $3 g$ & $2-\mathrm{NO}_{2}$ & $\mathrm{C}_{18} \mathrm{H}_{14} \mathrm{~N}_{3} \mathrm{O}_{3} \mathrm{SBr}$ & 431.9 & 190 & 72 & found & 49.99 & 3.21 & 9.70 \\
\hline & & & & & & calc. & 50.01 & 3.24 & 9.72 \\
\hline $3 h$ & $3-\mathrm{NO}_{2}$ & $\mathrm{C}_{18} \mathrm{H}_{14} \mathrm{~N}_{3} \mathrm{O}_{3} \mathrm{SBr}$ & 431.9 & 180 & 84 & found & 50.00 & 3.20 & 9.69 \\
\hline & & & & & & cal & 50.01 & 3.24 & 9.72 \\
\hline $3 \mathbf{i}$ & 6-Furyl & $\mathrm{C}_{16} \mathrm{H}_{13} \mathrm{~N}_{2} \mathrm{O}_{2} \mathrm{SBr}$ & 376.9 & 176 & 85 & Iound & 50.90 & 3.40 & 7.40 \\
\hline & & & & & & calc. & 50.94 & 3.44 & 7.42 \\
\hline $\mathbf{3 j}$ & $2-\mathrm{OH}$ & $\mathrm{C}_{18} \mathrm{H}_{15} \mathrm{~N}_{2} \mathrm{O}_{2} \mathrm{SBr}$ & 402.9 & 205 & 60 & found & 53.58 & 3.10 & 0.90 \\
\hline & & & & & & & 53.61 & 3.72 & 6.94 \\
\hline
\end{tabular}

\section{Spectral data of compounds $(3 \mathbf{a}-\mathbf{j})$}

4(4'-Bromophenyl)-6-(4"-methoxyphenyl)-1-acetyl-5, 6-dihydropyrimidine-2-thiol (3a)

IR (KBr) in $\mathrm{cm}^{-1}$ : 2550.11(S-H str), 1681.98 (N-C=O str), 1606.76 (C=C str), 1581.68(C=N str), 582.52 (Ar-Br str), 1114.89 (C-O-C str.); $\mathrm{H}^{1} \mathrm{NMR}\left(\mathrm{CDCl}_{3}\right) \delta(\mathrm{ppm}): 1.50(\delta, 1 \mathrm{H}, \mathrm{S}-\mathrm{H})$, $2.03\left(\delta, 3 \mathrm{H}, \mathrm{N}-\mathrm{CO}-\mathrm{CH}_{3}\right), 5.51\left(\mathrm{~d}, 1 \mathrm{H}, \mathrm{H}-\mathrm{C}_{6}\right.$-pyrimidine $), 6.73\left(\mathrm{~d}, 1 \mathrm{H}, \mathrm{H}-\mathrm{C}_{5}\right.$-pyrimidine), 7.17-7.66 (m, 8H, Ar-H).

4(4'-Bromophenyl)-6-(4"-hydroxy-3-methoxy-phenyl)-1-acetyl-5,6-dihydropyrimidine2-thiol (3b)

IR (KBr) in $\mathrm{cm}^{-1}: 2540.28$ ( $\mathrm{S}-\mathrm{H}$ str), 3450.25 (O-H str), 1712.85 (N-C=O str), 1589.40 $\left(\mathrm{C}=\mathrm{N}\right.$ str), 1599.04 (C=C str), 594.10 (Ar-Br str), 1074.39 (C-O-C str), $\mathrm{H}^{1} \mathrm{NMR}$ $\left(\mathrm{CDCl}_{3}\right) \delta(\mathrm{ppm}) ; 1.52(\delta, 1 \mathrm{H}, \mathrm{S}-\mathrm{H}), 2.03\left(\delta, 3 \mathrm{H}, \mathrm{NCOCH}_{3}\right), 2.11\left(\delta, 3 \mathrm{H}, \mathrm{C}-\mathrm{O}-\mathrm{CH}_{3}\right)$, $4.90(\delta, 1 \mathrm{H}, \mathrm{Ar}-\mathrm{OH}), 5.52\left(\mathrm{~d}, 1 \mathrm{H}, \mathrm{H}-\mathrm{C}_{6}\right.$ pyrimidine $), 6.74\left(\mathrm{~d}, 1 \mathrm{H}, \mathrm{H}-\mathrm{C}_{5}\right.$ pyrimidine $)$, 7.12-7.66 (m, 7H, Ar-H)

4(4'-Bromophenyl)-6-phenyl-1-acetyl,5,6-dihydro-pyrimidine-2-thiol (3c)

IR (KBr) in $\mathrm{cm}^{-1}: 2565.44$ ( $\mathrm{S}-\mathrm{H}$ str), 1689.97 (N-C=O str), 1606.76 (C=C str), 1585.64 $\left(\mathrm{C}=\mathrm{N}\right.$ str), $590.24(\mathrm{Ar}-\mathrm{Br}) ; \mathrm{H}^{1} \mathrm{NMR}\left(\mathrm{CDCl}_{3}\right) \delta(\mathrm{ppm}): 1.50(\delta, 1 \mathrm{H}, \mathrm{S}-\mathrm{H}), 2.04(\delta, 3 \mathrm{H}$, $\left.\mathrm{NCOCH}_{3}\right), 5.50\left(\mathrm{~d}, 1 \mathrm{H}, \mathrm{H}-\mathrm{C}_{6}\right.$ pyrimidine $), 6.72\left(\mathrm{~d}, 1 \mathrm{H}, \mathrm{H}-\mathrm{C}_{5}\right.$ pyrimidine $), 7.11-7.50$ (m, 9H, Ar-H). 
4(4'-Bromophenyl)-6-(4"-chlorophenyl)-1-acetyl 5,6-dihydropyrimidine-2-thiol (3d) IR (KBr) in $\mathrm{cm}^{-1}: 2542.38$ (S-H str), 1703.20 (N-C=O str), 1587.47 (C=C str), 1576.89 $\left(\mathrm{C}=\mathrm{N}\right.$ str), 619.17 (Ar-Br str), $750.01(\mathrm{Ar}-\mathrm{Cl} \mathrm{str}) ; \mathrm{H}^{1} \mathrm{NMR}\left(\mathrm{CDCl}_{3}\right) \delta(\mathrm{ppm}): 1.54(\delta, 1 \mathrm{H}, \mathrm{S}-\mathrm{H})$, $2.06\left(\delta, 3 \mathrm{H}, \mathrm{N}-\mathrm{CO}-\mathrm{CH}_{3}\right), 5.53\left(\mathrm{~d}, 1 \mathrm{H}, \mathrm{H}-\mathrm{C}_{6}\right.$ pyrimidine $), 6.72\left(\mathrm{~d}, 1 \mathrm{H}, \mathrm{H}-\mathrm{C}_{5}\right.$ pyrimidine), $7.15-7.60(\mathrm{~m}, 8 \mathrm{H}, \mathrm{Ar}-\mathrm{H})$.

4(4'-Bromophenyl)-6-(2'-chlorophenyl)-1-acetyl, 5,6-dihydropyrimidine-2-thiol (3e)

IR (KBr) in $\mathrm{cm}^{-1}: 2596.27$ ( $\mathrm{S}-\mathrm{H}$ str), $1681.98(\mathrm{~N}-\mathrm{C}=\mathrm{O}$ str), 1583.61 (C=C str), 1568.18 $\left(\mathrm{C}=\mathrm{N}\right.$ str), 513.08 (Ar-Br str), 702.11 (Ar-Cl str). $\mathrm{H}^{1} \mathrm{NMR}\left(\mathrm{CDCl}_{3}\right) \delta(\mathrm{ppm}): 1.56(\delta, 1 \mathrm{H}, \mathrm{S}-\mathrm{H})$, $2.08\left(\delta 3 \mathrm{H}, \mathrm{N}-\mathrm{CO}-\mathrm{CH}_{3}\right), 5.55\left(\mathrm{~d}, 1 \mathrm{H}, \mathrm{H}-\mathrm{C}_{6}\right.$ pyrimidine $), 6.73\left(\mathrm{~d}, 1 \mathrm{H}, \mathrm{H}-\mathrm{C}_{5}\right.$ pyrimidine), $7.16-7.62(\mathrm{~m}, 8 \mathrm{H}, \mathrm{Ar}-\mathrm{H})$.

4(4'-Bromophenyl)-6-(4"-dimethylaminophenyl)-1-acetyl-5,6-dihydropyrimidine-2-thiol (3f) IR ( $\mathrm{KBr})$ in $\mathrm{cm}^{-1}$ : 2548.05 (S-H str), 1680.99 (N-C=O str), 1583.61 (C=C str), 1508.38 (C=N str). $\mathrm{H}^{1} \mathrm{NMR}\left(\mathrm{CDCl}_{3}\right) \delta(\mathrm{ppm}): 1.57(\delta, 1 \mathrm{H}, \mathrm{S}-\mathrm{H}), 2.08\left(\delta, 3 \mathrm{H}, \mathrm{N}-\mathrm{COCH}_{3}\right), 3.03\left(\delta, 6 \mathrm{H}, \mathrm{N}\left(\mathrm{CH}_{3}\right)_{2}\right), 5.54$ (d, $1 \mathrm{H}, \mathrm{H}-\mathrm{C}_{6}$ pyrimidine), 6.74 (d, 1H, $\mathrm{H}-\mathrm{C}_{5}$ pyrimidine), 7.19-7.68 $(\mathrm{m}, 8 \mathrm{H}, \mathrm{Ar}-\mathrm{H})$.

4(4'-Bromophenyl)-6-(2'-nitrophenyl)-1-acetyl-5, 6-dihydropyrimidine-2-thiol (3g)

IR (KBr) in $\mathrm{cm}^{-1}$ : 2580.77 ( $\mathrm{S}-\mathrm{H}$ str), 1680.98 (N-C=O str), 1605.09 (C=C str), 1560.08 $\left(\mathrm{C}=\mathrm{N}\right.$ str), 560.02 (Ar-Br str), $1590.25\left(\right.$ Ar- $-\mathrm{NO}_{2}$ Asym str), 1340.22 (Ar- $\mathrm{NO}_{2}$ sym str). $\mathrm{H}^{1} \mathrm{NMR}\left(\mathrm{CDCl}_{3}\right) \delta(\mathrm{ppm}): 1.60(\delta, 1 \mathrm{H}, \mathrm{S}-\mathrm{H}), 2.10\left(\delta, 3 \mathrm{H}, \mathrm{N}-\mathrm{COCH}_{3}\right), 5.60\left(\mathrm{~d}, 1 \mathrm{H}, \mathrm{H}-\mathrm{C}_{6}\right.$ pyrimidine), 6.78 (d, $1 \mathrm{H}, \mathrm{H}-\mathrm{C}_{5}$ pyrimidine), 7.20-8.10 (m, $\left.8 \mathrm{H}, \mathrm{Ar}-\mathrm{H}\right)$.

4(4'-Bromophenyl)-6-(3'-nitrophenyl)-1-acetyl- 5,6 - dihydropyrimidine-2- thiol (3h)

IR (KBr) in $\mathrm{cm}^{-1}$ : 2580.77 (S-H str), 1680.90 (N-C=O str), 1605.08 (C=C str), 1560.04 (C=N str), 560.00 (Ar-Br str), 1540.00 (Ar- $\mathrm{NO}_{2}$ Asym. str), 1320.99 (Ar- $\mathrm{NO}_{2}$ sym. str). $\mathrm{H}^{1} \mathrm{NMR}\left(\mathrm{CDCl}_{3}\right) \delta(\mathrm{ppm}): 1.52(\delta, 1 \mathrm{H}, \mathrm{S}-\mathrm{H}), 2.05\left(\delta, 3 \mathrm{H}, \mathrm{N}-\mathrm{COCH}_{3}\right), 5.58\left(\mathrm{~d}, 1 \mathrm{H}, \mathrm{H}-\mathrm{C}_{6}\right.$ pyrimidine), $6.74\left(\mathrm{~d}, 1 \mathrm{H}, \mathrm{H}-\mathrm{C}_{5}\right.$ pyrimidine), 7.27-8.12 (m, 8H, Ar-H).

4(4'-Bromophenyl)-6-furyl-1-acetyl-5,6-dihydro- pyrimidine- 2-thiol (3i)

IR (KBr) in $\mathrm{cm}^{-1}: 2569.27$ ( $\mathrm{S}-\mathrm{H}$ str), 1683.91 ( $\mathrm{N}-\mathrm{C}=\mathrm{O}$ str), 1585.54 (C=C str), 1489.01 (C=N str), 569.02 (Ar-Br str), 1072.46 (Furyl ring C-O-C str), 2980.04 (C-H str), $\mathrm{H}^{1}$ NMR $\left(\mathrm{CDCl}_{3}\right) \delta(\mathrm{ppm}): 1.61(\delta, 1 \mathrm{H}, \mathrm{S}-\mathrm{H}), 2.10\left(\delta, 3 \mathrm{H}, \mathrm{N}-\mathrm{COCH}_{3}\right), 5.59\left(\mathrm{~d}, 1 \mathrm{H}, \mathrm{H}-\mathrm{C}_{6}\right.$ pyrimidine), 6.76 (d, $1 \mathrm{H}, \mathrm{H}-\mathrm{C}_{5}$ pyrimidine), 7.20-7.78 (m, 7H, Ar-H).

4(4'-Bromophenyl)-6-(2'-hydroxyphenyl)-1-acetyl-5,6-dihydropyrmidine-2-thiol (3j)

$\mathrm{IR}(\mathrm{KBr})$ in $\mathrm{cm}^{-1}$ : 2600.01 ( $\mathrm{S}-\mathrm{H}$ str), $1680.88(\mathrm{~N}-\mathrm{C}=\mathrm{O}$ str), $1585.55(\mathrm{C}=\mathrm{C}$ str $), 1568.18(\mathrm{C}=\mathrm{N}$ str), 599.88 (Ar-Br str), $3400.01(\mathrm{Ar}-\mathrm{OH}), \mathrm{H}^{1} \mathrm{NMR}\left(\mathrm{CDCl}_{3}\right) \delta(\mathrm{ppm}): 1.61(\delta, 1 \mathrm{H}, \mathrm{S}-\mathrm{H}), 2.09(\delta$, $\left.3 \mathrm{H}, \mathrm{N}-\mathrm{COCH}_{3}\right), 4.99(\delta, 1 \mathrm{H}, \mathrm{Ar}-\mathrm{OH}), 5.61\left(\mathrm{~d}, 1 \mathrm{H}, \mathrm{H}-\mathrm{C}_{6}\right.$ pyrimidine $), 6.75\left(\mathrm{~d}, 1 \mathrm{H}, \mathrm{H}-\mathrm{C}_{5}\right.$ pyrimidine), 7.18-7.78 (m, 8H, Ar-H).

Antimicrobial activity

The synthesized compounds (3a-j) were screened for their in vitro antimicrobial activity against, Bacillus subtilis, Escherichia coli, Klebsiella pneumoniae and Staphylococcus aureus and antifungal activity against, Aspergillus niger, Aspergillus flavus, Trichoderma viride, Candida albicans by measuring the zone of inhibition in $\mathrm{mm}$. The antimicrobial activity was performed by standard filter paper disc diffusion method ${ }^{16-17}$ and zone of inhibition is reported in the Table 3. Streptomycin and nystatin were used as standard drug for antibacterial and antifungal activities respectively. Nutrient agar was employed as culture medium and DMSO, was used as solvent. 
Table 2. Antimicrobial data of compounds 3a-j.

\begin{tabular}{|c|c|c|c|c|c|c|c|c|c|}
\hline \multirow[b]{2}{*}{$\begin{array}{l}\dot{0} \\
\dot{z}\end{array}$} & \multirow[b]{2}{*}{ Compounds } & \multicolumn{4}{|c|}{ Antibacterial } & \multicolumn{4}{|c|}{ Antifungal } \\
\hline & & $\begin{array}{l}\underset{\tilde{\Xi}}{\tilde{\Xi}} \\
\frac{\tilde{\sigma}}{\omega} \\
\infty\end{array}$ & $\underset{\substack{0 \\
0}}{\ddot{0}}$ & 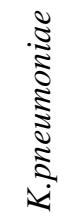 & 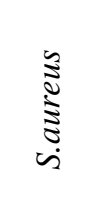 & 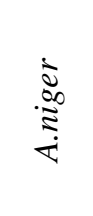 & 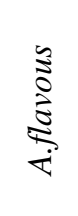 & 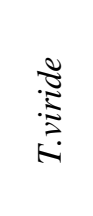 & 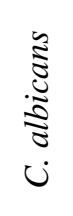 \\
\hline 1. & $3 \mathbf{a}$ & ++ & +++ & ++ & +++ & ++ & ++ & +++ & ++ \\
\hline 2. & $\mathbf{3 b}$ & ++ & ++ & ++ & ++++ & ++ & - & + & ++ \\
\hline 3. & 3c & ++ & ++ & ++ & +++ & ++ & + & ++ & - \\
\hline 4. & 3d & ++++ & - & ++ & ++ & + & + & ++ & + \\
\hline 5. & $3 e$ & + & + & ++ & + & ++ & + & ++ & - \\
\hline 6. & 3f & +++ & +++ & ++ & ++++ & ++ & ++ & ++ & + \\
\hline 7. & $3 g$ & ++ & + & - & + & +++ & +++ & +++ & +++ \\
\hline 8. & $3 \mathbf{h}$ & +++ & ++ & ++ & +++ & +++ & ++ & ++ & +++ \\
\hline 9. & $3 \mathbf{i}$ & +++ & +++ & ++ & +++ & ++ & +++ & +++ & +++ \\
\hline 10. & 3j & +++ & ++ & + & + & + & ++ & +++ & ++ \\
\hline Std. & Streptomycin & ++++ & +++ & +++ & ++++ & - & - & - & - \\
\hline drug & Nystatin & - & - & - & - & ++++ & +++ & ++++ & +++ \\
\hline
\end{tabular}

Zone of inhibition was measured in mm.++++: Strong activity, $+++:$ good activity, $++:$ moderate activity, + : poor activity, - : inactive.

In vitro anthelmintic activity ${ }^{18}$

In vitro anthelmintic screening studies of compounds 3a-j were performed by the Watkins technique, against common Indian earthworm 'P. posthuma'. For this purpose $4 \%$ and $2 \%$ solutions of the synthesized pyrimidine derivatives and standard drug piperazine hydrochloride in ethylene glycol were used for experiment. The experiments were performed in duplicate and average values of paralytic time and lethal time in minutes have given in the Table 3.

Table 3. Anthelmintic activity of compounds 3a-j (in minutes).

\begin{tabular}{cccccc}
\hline \multirow{2}{*}{$\begin{array}{c}\text { S. } \\
\text { No. }\end{array}$} & Compounds & \multicolumn{4}{c}{ Concentration, \% } \\
\cline { 3 - 6 } & & Paralytic time & Lethal time & Paralytic time & Lethal time \\
\cline { 3 - 6 } & $\mathbf{3 a}$ & 7 & 15 & 9 & 18 \\
2. & $\mathbf{3 b}$ & 8 & 17 & 8 & 20 \\
3. & $\mathbf{3 c}$ & 10 & 18 & 10 & 21 \\
4. & $\mathbf{3 d}$ & 9 & 17 & 9 & 17 \\
5. & $\mathbf{3 e}$ & 6 & 14 & 7 & 17 \\
6. & $\mathbf{3 f}$ & 8 & 16 & 7 & 16 \\
7. & $\mathbf{3 g}$ & 9 & 15 & 10 & 20 \\
8. & $\mathbf{3 h}$ & 9 & 17 & 9 & 18 \\
9. & $\mathbf{3 i}$ & 8 & 15 & 9 & 17 \\
10. & $\mathbf{3 j}$ & 7 & 14 & 10 & 18 \\
11. & Std. drug & 6 & 13 & 7 & 15 \\
\hline
\end{tabular}




\section{Insecticidal activity ${ }^{19-20}$}

Adult cockroaches ( $P$. americana) were selected for the testing of, in vitro insecticidal activity. $4 \%$ solutions of synthesized pyrimidine derivatives and standard drug cypermethrin $(w / v)$, in acetone were used for experiment. The time of death of cockroaches was noted, on an average and denoted as KD (Knock Down) value in minutes. For each sample three replication were performed and same experiments were performed with standard drug.

Table 4. Insecticidal activity of compounds 3a-j (in minutes).

\begin{tabular}{cccc}
\hline S. No. & Compounds & $\mathrm{R}^{2}$ & K.D. Value, min \\
\hline 1. & $\mathbf{3 a}$ & $4-\mathrm{O}-\mathrm{CH}_{3}$ & 9 \\
2. & $\mathbf{3 b}$ & $4-\mathrm{OH}-3-\mathrm{OCH}_{3}$ & 9 \\
3. & $\mathbf{3 c}$ & $\mathrm{H}$ & 10 \\
4. & $\mathbf{3 d}$ & $4-\mathrm{Cl}$ & 12 \\
5. & $\mathbf{3 e}$ & $2-\mathrm{Cl}$ & 8 \\
6. & $\mathbf{3 f}$ & $\left.4-\mathrm{N}^{-} \mathrm{CH}_{3}\right)_{2}$ & 8 \\
7. & $\mathbf{3 g}$ & $2-\mathrm{NO}_{2}$ & 7 \\
8. & $\mathbf{3 h}$ & $3-\mathrm{NO}_{2}$ & 9 \\
9. & $\mathbf{3 i}$ & 6-Furyl & 7 \\
10. & $\mathbf{3 j}$ & 2-OH & 9 \\
11. & Std. drug & Cypermethrin & 8 \\
\hline
\end{tabular}

\section{Conclusion}

All the synthesized pyrimidine-2-thiols $\mathbf{3}(\mathbf{a}-\mathbf{j})$ have given appreciable yield with satisfactory elemental analysis. It is inferred from the Table $2,3 \& 4$, that synthesized compounds $\mathbf{3 a - j}$, have shown significant antibacterial activity, good to moderate antifungal activity and significant anthelmintic and insecticidal activities, due to the presence of thio group, which enhance the respective activities with varied substituent groups, against selected pathogens and organisms.

\section{Acknowledgment}

Authors wish to express their thanks \& gratitude to the Head of the Department, Chemistry, Dr. H.S. Gour University, Sagar, for providing necessary facilities, Prof. Archna Mehta \& Prof. P. Mehta, Dept. of Botany, Prof. M. Bhide Dept. of Zoology, for their help and guidance to carry out biological activities and Director, CDRI, Lucknow, for providing spectral data.

\section{References}

1. Salahuddin Md, Kakad Sunil and Shantakumar S M, E Journal of Chemistry, 2009, 6(3), 801-808.

2. Heda L C, Sharma Rashmi, Pareek C and Chaudhari P B, E Journal of Chemistry, 2009, 6(3), 770-774.

3. Jain Vivek Kumar and Rao J T, J Inst Chem India, 2003, 75(1), 24-26.

4. Mohammad Amir, Aggrawal R and Javed S A, Oriental J of Chem., 2004, 20(3), 477-480.

5. Jing-Jong Lu and Herbert L W, Chem Abstr., 1986, 105, 97462z.

6. Zhichkin Pavel, Fairfax D J and Eisenbeis S A, Synthesis, 2002, 6, 695.

7. Quiroga Jairo, Insuasty B, Hormaza A, Gamenara D, Dominguez L and Saldana J, $J$ Heterocycl Chem., 2009, 36(1), 11-13. 
8. Rahatgaonkar Anjali Milind and Ghiya B J, Asian J Chem., 1998, 10(4), 958-963.

9. Devries E G E, Gietma J A, Workman P, Scott J E, Craw Shaw A, Dobbis H J, Dennis I, Mulder N, Sleijfer D T H and Willemse P H B, Br J Cancer, 1993, 68, 661.

10. Gangiee A, Zeng Y, McGuire J J, Kisliuk R L, J Med Chem., 2000, 43, 3125.

11. Alagarsamy Veerachamy, Shankar Durairaj and Solomon Viswas Raja, ARKIVOC, 2006, 16, 149-159.

12. Nega S, Aionso J, Diazj A and Junquere F, J Heterocycl Chem., 1990, 27, 269.

13. Horwitz J P and Chua Noel M, J Org Chem., 1964, 29, 2076.

14. Kappe C O, Eur J Med Chem., 2000, 35, 1043.

15. Pulman A David, Smith H Ian, Larkin P John and Casida E John, Pestic Sci., 1999, 46(3), 237-245.

16. Safary A, Motamedi H, Maleki S and Sevvednejad, Int J Botany, 2009, 5(2), 176-180, doi: 10.3923/ijb.2009.176.180.

17. Bhattacharjee, Paul Satya B, Nath Abhijit, Choudhury P P N and Choudhury Sudip Materials, 2009, 2, 345-352.

18. British Pharmacopedia, General Medical Council, London, 1958, 1078, 600.

19. Wrignt Brain J, Baillie Alister C and Dowsett R John, Pestic Sci., 2006, 8(4), 323-330.

20. Kagabu Shinzo, Ogama Mikako, Makimura Masayuki and Nishimura Keiichiro, $J$ Pestic Sci., 2005, 30(1), 44-46. 


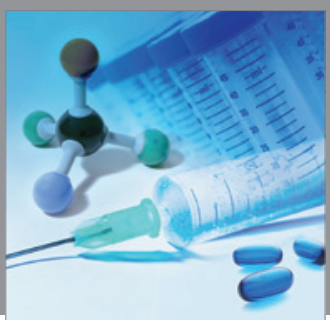

International Journal of

Medicinal Chemistry

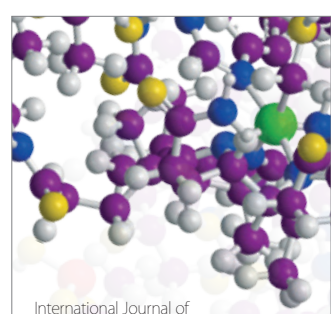

Carbohydrate Chemistry

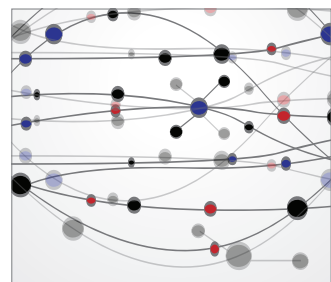

The Scientific World Journal
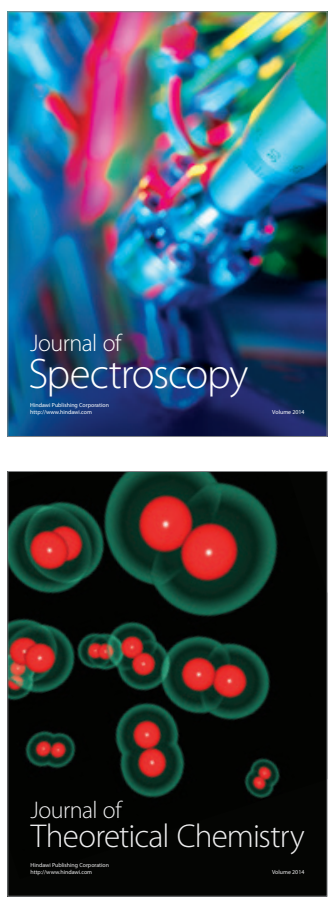
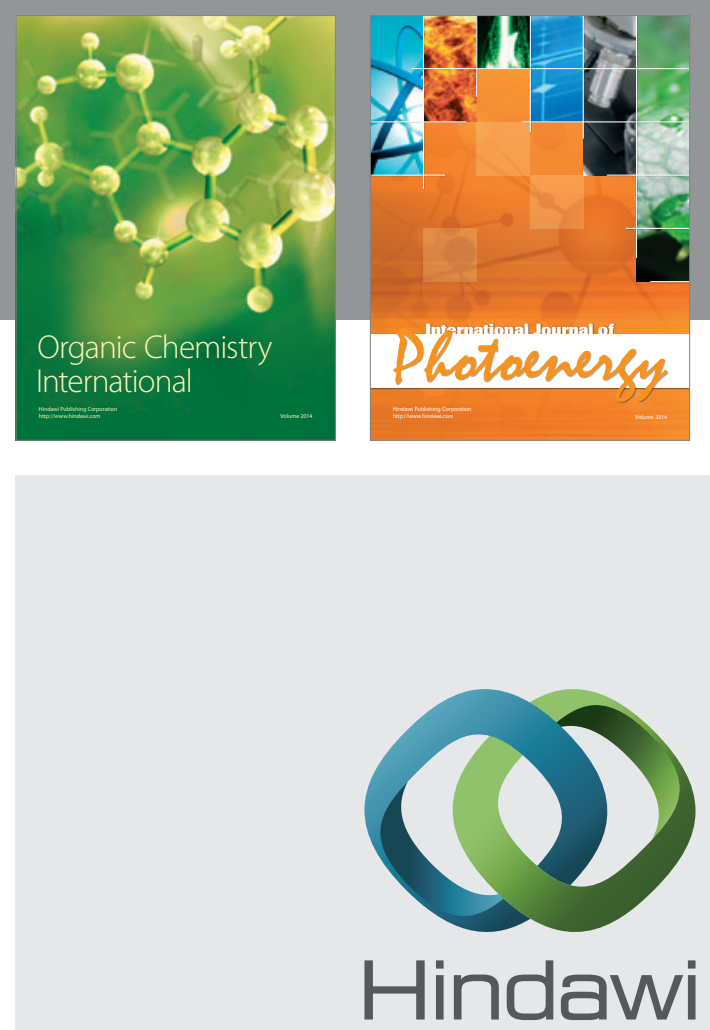

Submit your manuscripts at

http://www.hindawi.com
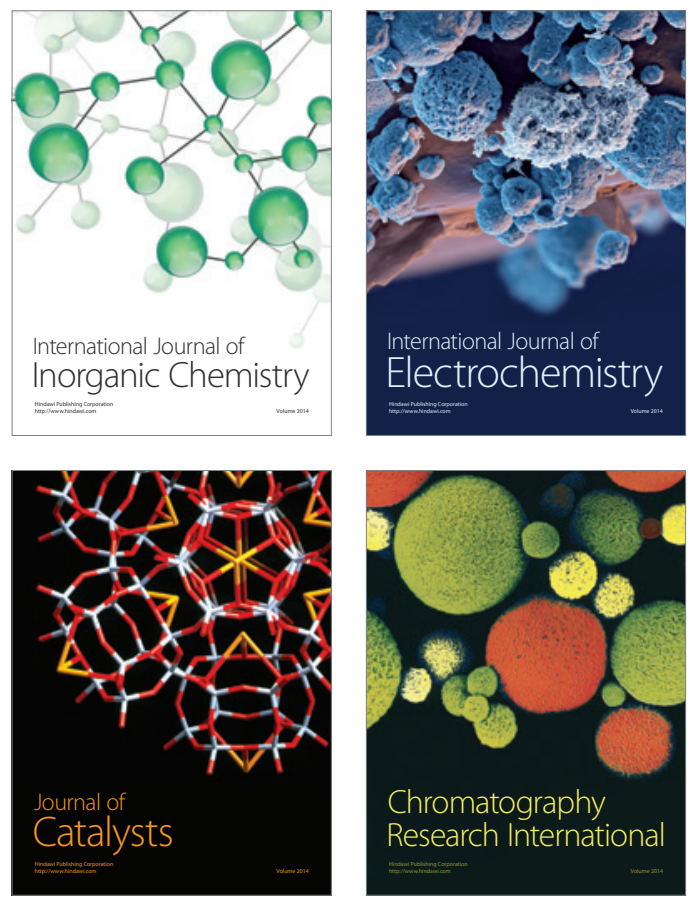
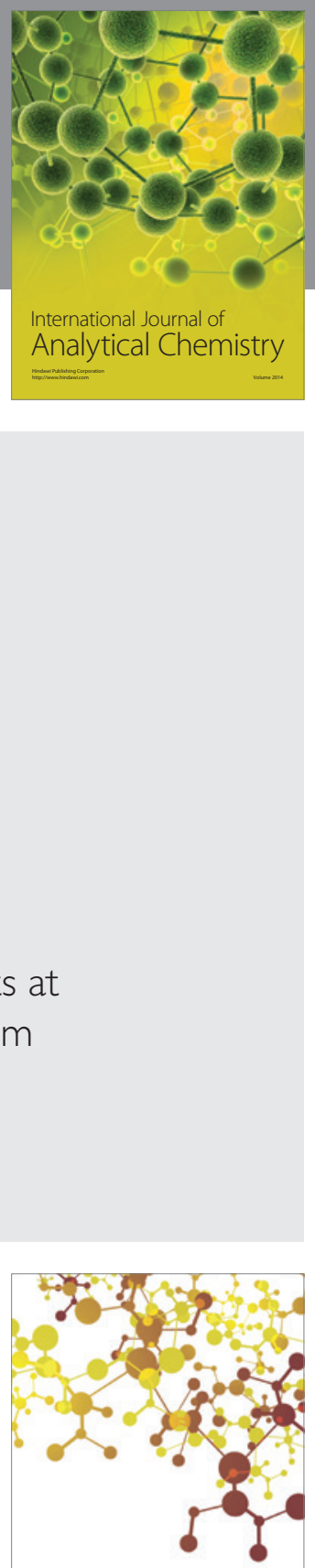

Journal of

Applied Chemistry
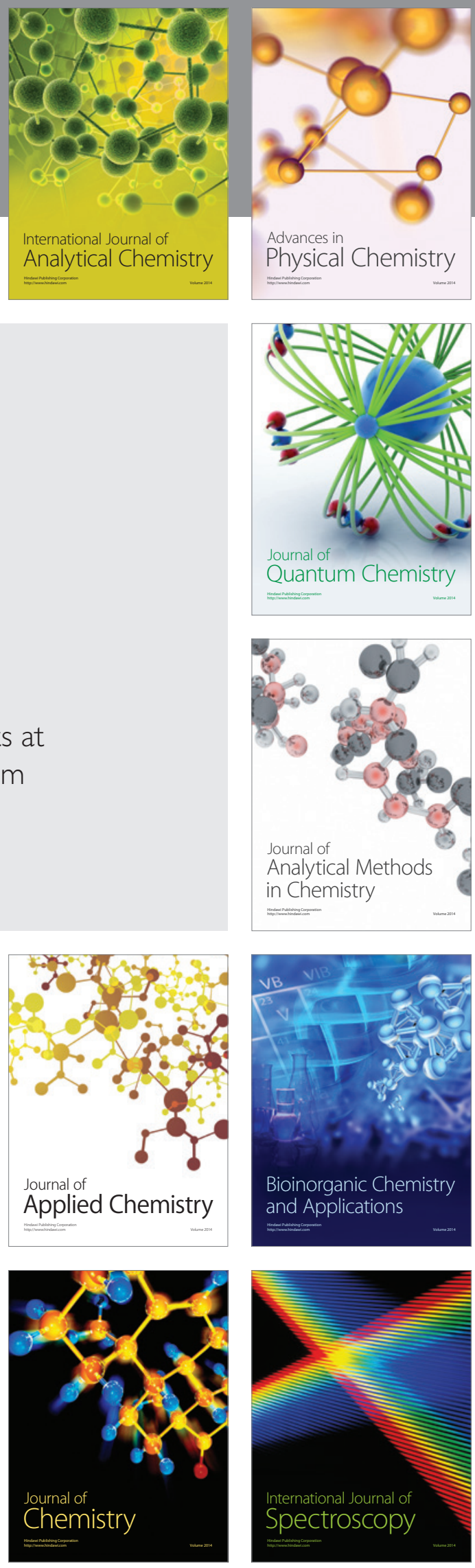